très prononcée le long du bord postérieur, limitée de chaque côté par un léger repli tuberculiforme qui se prolonge en un petit triangle saillant, surmontant chaque racine de la pince. Bord postérieur épais et légèrement concave entre les branches de la pince.

Segoments inférieurs plus clairs, d'un brun jaunâtre, ponctués. Pénultième segment ventral rugueux et grossièrement ponctué; Pygidium non saillant, vertical, échancré supérieurement.

Branches de la pince d'un fauve ferrugineux, écartées à la base, robustes et légèrement dilatées à leur naissance, triquètres en dessus dans leur premier tiers avec une impression très marquée contre la racine à droite et à gauche de l'arête médiane, plates en dessous, puis cylindriques, elles vont s'amincissant jusqu'aux pointes aiguës qui se touchent. D'abord droites, elles se courbent un peu au delà de leur moitié, la droite plus sensiblement que la gauche, l'ensemble de leurs arêtes internes formant un ovale. Bord interne lisse.

O : Segments de l'abdomen moins anguleux sur les côtés du $3^{\mathrm{e}}$ au $7^{\mathrm{e}}$ et dépourvus de carènes latérales. Dernier segment dorsal plus rétréci postérieurement, sans dépression transversale, avec les replis moins accusés.

Branches de la pince marron rougeâtre, plus claires que l'abdomen, presque contiguës, robustes et triquètres pour plus d'un tiers de leur longueur, puis elles s'amincissent et s'arrondissent sensiblement jusqu'aux pointes aiguës qui s'entre-croisent; subdroites, modérément courbées seulement un peu avant les pointes. Bord interne crénelé dans sa première moitié, puis lisse.

Longueur totale du corps : $\sigma^{\top}, 16$ millimètres; 9 , 18 millim. 5.

Longueur des branches de la pince : $\sigma^{7}$, à droite, 2 millim. 3 ; à gauche, 2 millim. 5; ㅇ, 2 millim. 2.

$1 \sigma^{x}$ de Troya, $1901, D^{r} G$. Rivet; 1 कt $1 \sigma^{x}$ juv. ainsi que 2 larves de El Pelado, 19o3, Dr G. Rivet.

\title{
UNE NOUVELLE ESPÈCE
}

de Promachocrinos (Promachocrinus Joubini), PAR C. VANEY.

L'expédition du $D^{r}$ Charcot a rapporté de la baie de Biscoë, par $64^{\circ}$ de latitude sud, une nouvelle espèce de crinoïde appartenant à ce curieux genre à dix radius, le genre Promachocrinus. Je suis heureux de dédier cette nouvelle espèce à M. le $\mathrm{D}^{\mathrm{r}}$ Joubin, Professeur au Muséum d'histoire naturelle de Paris, en reconnaissance de son extrême oblig̣eance.

L'unique exemplaire est étalé et mesure 200 millimètres de diamètre; 
sa couleur est marron jaunâtre; les cirres sont brunâtres et les différentes articulations se détachent du fond par leur coloration blanchâtre.

Les cirres sont au nombre d'une cinquantaine, disposés plus ou moins irrégulièrement en quatre ou cinq étages sur une centro-dorsale en forme de cône surbaissé, dont la hauteur est de 5 millimètres et le diamètre de base 6 millimètres. L'apex de la centro-dorsale est dépourvu de cirres sur une surface circulaire de 4 millimètres de diamètre. Quelques cirres sont en voie de régénération, la plupart ont une longueur de 60 millimètres et sont

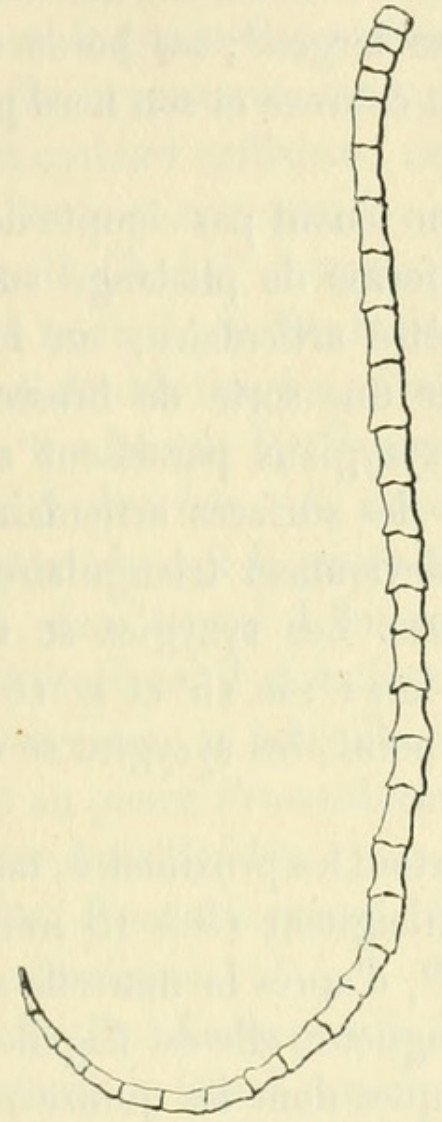

Fig. 1.

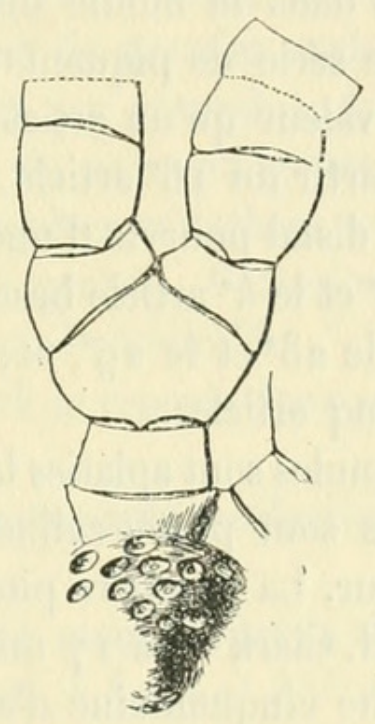

Fig. 2.

composés d'une trentaine d'articles (fig. 1). Les trois articles proximaux ont la forme de disques un peu plus larges que hauts; le quatrième article a un diamètre sensiblement égal à la hauteur et tous les autres articles ont la forme d'osselets de phalange, dont la longueur est égale à une fois et demiele diamètre; dans les huit derniers articles distaux les dimensions vont en s'atténuant progressivement; le dernier article se termine en pointe.

Les radiales, au nombre de dix, sont bien visibles; elles sont soudées entre elles sur toute la longueur de leurs faces latérales contiguës; leur bord distal est légèrement concave et enchâsse la première costale; celle-ci a la forme d'un disque dont la hauteur est égale à la moitié de la largeur (fig. 2). Ces premières costales sont presque toutes indépendantes les unes des autres, cependant quelques-unes sont faiblement en contact entre elles par leurs bases. 
La costale axillaire est grande et losangique; sa largeur est de 5 millimètres environ, alors que sa hauteur n'est que de 3 millim. 2 ; son bord proximal est légèrement saillant dans sa partie médiane, tandis que ses faces articulaires distales sont presque rectilignes.

Les bras sont au nombre de vingt et comprennent chacun plus de 150 segments. La première brachiale a la forme d'un disque aplati, à bord distal légèrement échancré, dont la hauteur est égale à la moitié du diamètre de base; les deux premières brachiales d'un même radius ont leurs bases en contact l'une avec l'autre par leur côté interne. La deuxième brachiale a une hauteur sensiblement égale à sa largeur; ses bords latéraux sont légèrement convexes, son bord distal est convexe et son bord proximal offre une pointe articulaire saillante.

Dans un premier examen superficiel, en ne tenant pas compte des syzygies, les quinze articles suivants ont la forme de phalange surbaissée dont la hauteur est égale aux 3/4 du diamètre articulaire; sur leur face dorsale et dans la moitié distale, fait saillie une sorte de brosse constituée d'une série de piquants; deux articles syzygiaux paraissent avoir ici la même valeur qu'un article n'offrant que des surfaces articulaires obliques. A partir dn $16^{\mathrm{e}}$ article, les segments deviennent triangulaires et ont leur bord distal pourvu d'une brosse épineuse. Les syzygies se trouvent entre le $3^{\circ}$ et le $4^{\circ}$ article brachial, le $10^{\circ}$ et le $11^{\circ}$; le $15^{\circ}$ et le $16^{\circ}$, le $22^{\circ}$ et le ${ }_{2} 3^{\mathrm{e}}$, le ${ }_{2} 3^{\mathrm{e}}$ et le $29^{\mathrm{e}}$, et, à partir de ce point, les syzygies se répètent tous les cinq articles.

Les pinnules sont aplaties latéralement, surtout les proximales, tandis que les distales sont plutôt cylindriques; elles atteignent 15 à 18 millimètres de longueur. La première pinnule externe $\left(\mathrm{P}_{1}\right.$ d'après la nouvelle notation d'Austin H. Clark (') ) a 17 millimètres de longueur; elle est flagellée, composée d'une cinquantaine d'articles cylindriques dont les quinze premiers portent des piquants latéro-ventraux. La deuxième pinnule externe $\left(\mathrm{P}_{2}\right)$ est plus courte, plus massive et ne possède que 15 segments. La troisième pinnule externe $\left(\mathrm{P}_{3}\right)$ a une vinglaine d'articles et se termine en flagelle.

La première pinnule interne $\left(\mathrm{P}_{a}\right)$ est courte et a 15 segments; la deuxième $\left(\mathrm{P}_{b}\right)$ est longue, flagellée et a 20 segments, la troisième pinnule $\left(\mathrm{P}_{c}\right)$ a 18 segments. Les pinnules distales sont cylindriques et se composent d'une vingtaine d'articles allongés, à région moyenne évidée et à bord distal quelquefois armé d'un petit nombre de piquants. Le long des articles, les saccules sont brunâtres et de grande taille, au nombre de cinq à six par segment. Sur les aires ambulacraires du disque, des bras et des pinnules, on ne distingue pas de plaquettes ambulacraires. Les pédicelles sont cylindriques.

(1) Austin H. Cuark. New Genera and species of Crinoids. Proceedings of the Biological Society of Washington, 1908, vol. XXI, p. 220. 
Rapports et différences. - En 1879 , Carpenter ${ }^{(1)}$ établit le genre Promachocrinus pour toutes les espèces de Crinoïdes à dix radius; il rattacha ce nouveau genre aux Comatulidés. Bather $(1900)^{(2)}$ considère ces formes à dix radius comme des variations méristiques permanentes.

En 1905 , Minckert ${ }^{(3)}$ établit pour ces Crinoïdes à dix radius la famille des Décamétrocrinidés et démembra en deux l'unique genre de Carpenter. Il rangea dans un nouveau genre Decametrocrinus les espèces à dix bras primaires où il n'existe pas d'axillaire; toutes ces formes sont de grande profondeur et possèdent une centro-dorsale petite et aplatie. Minckert ne laissa dans le genre Promachocrinus emend que les espèces, à vie littorale, qui ont dix rayons avec vingt bras secondaires, par suite chez lesquelles existe des costales axillaires; ces formes ont toutes une centro-dorsale très grande, haute et conique.

Austin H. Clark ${ }^{(4)}$ montre avec justesse que cette nouvelle famille de Grinoïdes ne doit pas être maintenue, car les formes à dix radius semblent avoir une double origine : les Promachocrinus ressemblent beaucoup aux Antedon, tandis que les Decametrocrinus offrent de grandes analogies avec certaines Eudiocrinus (E. atlanticus E. Perrier, par exemple). Aussi A. H. Clark rapproche-t-il le genre Decametrocrinus du nouveau genre Pentametrocrinus, dans lequel il fait entrer certaines espèces rattachées autrefois au genre Eudiocrinus. Il constitue, avec ces deux genres, Decametrocrinus et Pentametrocrinus, la nouvelle famille des Pentamétrocrinidés.

Quant au genre Promachocrinus, A. H. Clark le reporte dans sa nouvelle famille des Antedonidés.

Les deux Promachocrinus déjà décrits appartiennent exclusivement à la région antarctique.

L'un, le Pr. Kerguelensis Carpenter, a été recueilli par le Challenger. sur le littoral de l'ìle Kerguelen et de l'ìle Head, à $52^{\circ} 59^{\prime} 3 o^{\prime \prime}$ de latitude sud et par une profondeur de 18 à 231 mètres. La Discovery l'a recueilli à nouveau à des profondeurs de 100 brasses ${ }^{(5)}$.

L'autre, le Pr. Vanhöffenianus Minckert, a été rapporté par le Gauss qui l'a récolté par 350 à 400 mètres de profondeur dans la zone littorale de terres antarctiques situées à $66^{\circ} 2^{\prime} 9^{\prime \prime}$ de latitude sud.

La découverte du Pr. Joubini par le Français accentue ce fait que le genre Promachocrinus est localisé dans l'Antarctique.

(1) Proc. Boy. Soc., 1879 , XXVII, p. 385.

(2) A Treatise on Zoology, III, Echinodermata, p. $19^{5}$.

(3) Das genus Promachocrinus, zugleich ein Beitrag zur Faunistik der Antarktis. Zool Anz., Bd. XXVIII, p. 490.

(4) A.H. Cилвк. New genera of unstalked Crinoids. Proceed. Biol. Soc. Washington, XXI, 1908.

(5) Bell. Echinoderma, Natural antartic Expedition, Natural History, Vol. IV, p. 3. PI. I. 
Le Pr. Joubini se rapproche plutôt du Pr. Kerğuelensis que du Pr. Vanhöffenianus. Ce dernier possède, en effet, un squelette ambulacraire formé de plaquettes qui n'existe ni chez le Pr. Kerguelensis ni chez le Pr. Joubini; d'ailleurs, chez ces deux dernières espèces, la costale axillaire est rhombique, alors que, chez le $\operatorname{Pr}$. Vanhöffenianus, cette axillaire a, comme la deuxième brachiale, une allure grêle. Si nous nous en rapportons aux figures données soit par Carpenter, soit plus récemment par Bell, l'axillaire parait plus net-tement losangique chez le $\mathrm{Pr}$. Joubini que chez le $\mathrm{Pr}$. Kerguelensis. Les premiers articles des bras ont, chez le $\mathrm{Pr}$. Joubini, une forme bien particulière avec cette brosse de piquants dorsaux. La disposition des syzygies, la structure des premières pinnules n'est d'ailleurs pas la même dans ces deux espèces, qui se séparent nettement l'une de l'autre par ce fait que l'apex de la centro-dorsale est nu chez le Pr. Joubini alors qu'il est pourvu de cirres chez le Pr. Kerguelensis.

Les cirres du Pr. Joubini sont assez comparables à ceux du Pr. Vanhöffenianus; en effet, dans ces deux espèces, ils présentent à leur base trois ou quatre articles courts, tandis que les autres segments des cirres sont allongés.

\section{Les Ombellifères de la mission Pelliot-Vatllant,}

par M. H. de Boissieu.

J'ai étudié dernièrement les Ombellifères de la belle collection de plantes rapportée par MM. Pelliot et Vaillant de leur mission en Asie centrale. Cette collection comporte, pour la famille que j'examinais, seulement treize numéros. Mais la plupart de ces numéros offrent un intérêt réel. Outre deux Ombellifères complètement nouvelles pour la science, auxquelles j'ai donné le nom des deux chefs de mission, la collection comprenait deux Ombellifères mal connues, dont une nouvelle pour l'herbier du Muséum de Paris. En outre, les matériaux rapportés par MM. Pelliot et Vaillant permettent de constater un fait qu'on pressentait après les travaux de Diels, la Flore de Corée de Nakaï, etc., à savoir, la grande affinité de de la Flore himalayenne et de la Flore des montagnes de l'extrême nord de la Chine, au moins pour certains genres, Bupleurum, Pituranthos, etc.

Les découvertes les plus curieuses de MM. Pelliot et Vaillant, à en juger par la seule famille des Ombellifères, semblent avoir été faites dans les explorations autour de Ku-Tchar, dans le Turkestan chinois. Les plantes de cette région paraissent se signaler par leur aspect d'herbes désertiques. En ce qui regarde les Ombellifères, les feuilles, presque toutes basilaires, ont le limbe extrêmement découpé en lanières linéaires, de manière que la surface d'évaporation soit le plus réduite possible : les gaines des vieilles 


\section{$2 \mathrm{BHL}$ Biodiversity Heritage Library}

1910. "Une nouvelle espece de Promachocrinus." Bulletin du Muse

um national d'histoire naturelle 1910, 158-162.

https://doi.org/10.5962/bhl.part.20423.

View This Item Online: https://www.biodiversitylibrary.org/item/27184

DOI: https://doi.org/10.5962/bhl.part.20423

Permalink: https://www.biodiversitylibrary.org/partpdf/20423

\section{Holding Institution}

New York Botanical Garden, LuEsther T. Mertz Library

\section{Sponsored by}

MSN

\section{Copyright \& Reuse}

Copyright Status: NOT_IN_COPYRIGHT

This document was created from content at the Biodiversity Heritage Library, the world's largest open access digital library for biodiversity literature and archives. Visit BHL at https://www.biodiversitylibrary.org. 